\title{
The development of an ontology model for early identification of children with specific learning disabilities
}

\author{
Rosmayati Mohemad, Nur Fadila Akma Mamat, Noor Maizura Mohamad Noor, Arifah Che Alhadi \\ Faculty of Ocean Engineering Technology and Informatics, Universiti Malaysia Terengganu, Malaysia
}

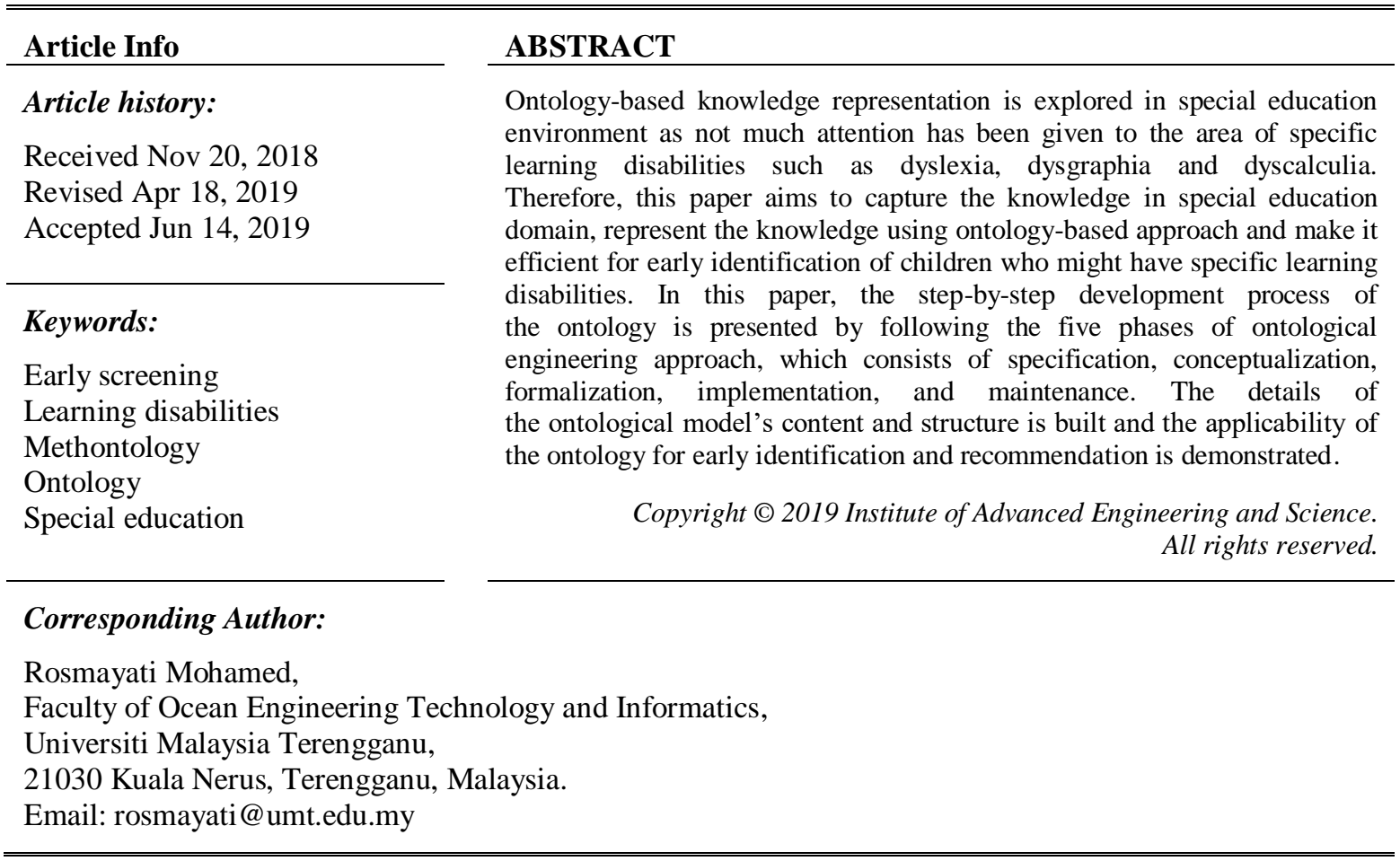

\section{INTRODUCTION}

The increasing use of knowledge-based technology nowadays is due to the demands for the complete access to useful information, which is often implicit, physically distributed and heterogeneous in structure, content and source [1]. The effective use of the meaningful information is vital for supporting decision-making and solving complex problems. Development of knowledge-based systems in a number of specific domains such as management, business, biological sciences, medical, education and others has led decision makers and the public to utilize the captured human's expert knowledge in decision-making process [2,3]. Therefore, the need to explicitly represent the knowledge in a formal way is important in the development of knowledge-based systems, with respect to express knowledge for completing more meaningful tasks.

Ontology is one of the technology designed to semantically specify the concepts and relationships of a domain knowledge and represent this knowledge in a structured and explicit way, which can be exploited by computer programs $[4,5]$. This complex and rich knowledge are described as facts and rules. It can be obtained from human's experiences and expertise, as well as documents and reports. In this sense, ontology is useful for the purpose of enabling the sharing of common understanding on particular knowledge, the re-use of domain knowledge and the better engineering of knowledge based systems, which allow automatic reasoning and interpretation through the body of knowledge [6].

In education, many ontology-based applications have been developed to effectively overcome the barriers of sharing information across educational applications [7], teaching problem solving [8], providing frameworks for learning outcomes reuse [9, 10] and enabling intelligent and personalized student support $[11,12]$. Furthermore, ontology also has been adopted in special education domain to identify types of special education $[2,13]$ and recommend suitable teaching and learning process according to the special needs 
of the special student [14-17]. However, not much attention has been given to the area of specific learning disabilities such as dyslexia, dysgraphia and dyscalculia.

Early identification of children who may at risk of having specific learning disabilities is crucial in order to assist them in overcoming barriers to learn. As the consequences, this will helps to prevent the growth of child with academic failure, lower motivation, greater frustration, distress, school dropout, and peers rejection $[18,19]$. This process, however could be extremely complicated as the identification itself is highly depends on human experts such as medical practitioners, occupational therapists and special education teachers as it involves a series of thorough screening and diagnosis activities that need to be done manually, hence leading to increased manpower and time consumption [20,21]. Currently, there is no comprehensive and universally accepted standards for the early identification of children with learning problems. Thus, early identification of children who may at risk of having specific learning disabilities by general users such as parents and teachers is vital for the early intervention.

Therefore, ontology is one of the technological advancements that can be used to structure the knowledge in a standard machine-readable format, so that the data will be understandable and analyzable by the computers. The objective of this paper is to build an ontology model for early identification of children with specific learning disabilities. The rest of the paper is organized as follows. Section 2 presents the overall framework of the research method.The process of building the proposed ontology is discussed in Section 3. Finally, the last section concludes with a summary of this paper and future research directions.

\section{RESEARCH METHOD}

The proposed ontology model is built by acquiring information from a series of domain expert interviews. Comprehensive reviews on the related researches are also important to identify the purposes and concrete justification for engineering the ontology model. The ontology model's static knowledge and dynamic knowledge are formulated to define the concepts, semantic relationships, attributes, formal axioms and logic rules. Figure 1 shows the overall phases in the ontology development including specification, conceptualization, formalization and implementation, maintenance and documentation.

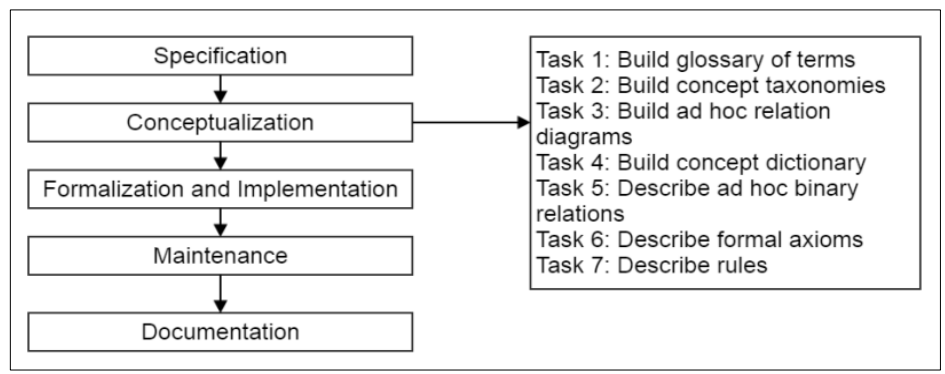

Figure 1. The phases for developing ontology model

\subsection{Specification}

This phase aims to determine the purposes and scopes of developing the ontology. It also includes feasibility studies which were carried out to target the potential platforms and applications where the ontology will be used and integrated. This process is vital to justify either ontology model is possible and suitable to be built for solving particular problems. There are several research methods that have been conducted in this process:

a. Review of existing literatures in journal articles, conference papers, technical reports, thesis or dissertation and other documents related to educations and special education domains. Next, the analysis is downsized to focus on the specific learning disability with respect to solve the main issues related to three types of specific learning disabilities; dyslexia, dysgraphia and dyscalculia.

b. Analysis of several existing ontology models in education domain for reusability purposes. It includes the process on searching for existing ontologies using semantic web search engine platform such as Swoogle and standard search engine such as Google. The existing ontologies also are searched in related ontology journals and other scientific publications.

c. Series of unstructured interviews with domain experts in specific learning disabilities such as medical practitioners, special education teachers and occupational therapist, and also interviews with potential users such as parents of children with specific learning disabilities and school teacher. 
At this process, it answers a set of competency questions to allow relevant and precise knowledge representation in ontology. Middle-out approach was used to build a set of competency questions. Initially, a list of basic questions is formed, then the results of these questions enables a more specific questions to be raised. Ontology developers verify the procedure used in determining the requirements.

\subsection{Conceptualization}

Conceptualization relates to the organization of knowledge acquired during the knowledge acquisition activities using external representation, which is independent of the knowledge representation paradigms and implemented languages in which the ontology will be formalized and implemented. Conceptual design is the most important process in the development of an ontology in which it maps relevant information into a conceptual model. It advances from the knowledge level to the implementation level. In conceptualization, the knowledge obtained in the specification level are structured into concepts or classes, attributes, relationships, formal axioms, rules and individuals or instances (if any). The related general terms in special education are reused from the existing ontologies (if any) before specific terminologies to application domain is expanded. Based on METHONTOLOGY approach, conceptualization which consists of seven (7) tasks is detailed out as follows:

a. Building a set of glossaries of terms including concepts, properties and relationships between concepts that related to special education domain. The natural language description for each term is defined along with its synonyms and acronyms terms. Output of this task is a documentation of glossary of terms.

b. Defining the concept taxonomies by classifying the concepts based on their inheritance relationships. The classification of inheritance relationships between super concept (super class) and sub-concepts (subclass) is designed in hierarchical structure. There are four types of inheritance relationships to classify the concepts; subclass-of, disjoint-decomposition, exhaustive-decomposition and partition. At the end of this task, a domain taxonomy of all related concepts is built.

c. Identifying ad hoc binary relationships between concepts and representing them in semantic network diagrams. The results obtained from this task is a set of ad hoc binary relationship diagrams.

d. Specifying properties and relationships that describe each of the concept taxonomies and ad hoc binary relationships in a concept dictionary. Output from this task is a table of concept dictionaries.

e. Describing in detail all the ad hoc binary relations included in the concept dictionary. The result of this task is the ad hoc binary relation table.

f. Describing in detail the formal axioms. In this task, necessary class axioms are identified and represented in formal logical expression which is built for the purpose of knowledge reasoning. The finding of this task is a set of formal axioms table.

g. Describing rules to infer new knowledge based on the patterns of existing knowledge. A set of rules are identified and described in a rule table. These rules are expressed and realized using SPARQL.

\subsection{Formalization and implementation}

In this phase, the formalization activity transforms the conceptual model into a formal model. There are many languages that can be used for encoding the conceptual model such as RDF, RDFS, XML, OIL, DAML+OIL, OWL, OWL2 and OWL-Full for the purpose of making the model is readable by the computer program. In this research, the conceptual ontology is developed using OWL, RDF and RDFS in which the selection of theses languages are based on the suitability of the language compile with ontology editor that was used. There are several ontology editors that can be used such as Protégé, SWOOP, NeOn Toolkit, TopBraid Composer, and others. These tool allows to specify concepts in a class hierarchy and provide the description of the properties, constraints and relations with others concept. In this research, TopBraid Composer is used as the ontology editor tool.

\subsection{Maintenance}

Maintenance is another important process in the development of an ontology in order to ensure the ontology is current and comprehensive. In this phase, it involves the activities of updating and correcting ontology model and its documentation based on post-development feedbacks obtained from a series of interviews with domain experts. Besides, an ontology-based prototype is used to check the ontology model either it is correctly modeled the knowledge or not in order to support specific task.

\subsection{Documentation}

Identified objectives, scopes, processes, concepts, relations, properties, axioms and rules are recorded in a complete documentation. Documentation is vital to explicitly explain the ontology taxonomies, thus facilitating other ontology developers to refer. Therefore, the developed ontology can be shared and expand for other purposes. 


\section{RESULTS AND DISCUSSION}

The focus of this paper is the building of the ontology model that capables to early identify children with potential to have specific learning disabilities.

\subsection{Determine competency questions}

In this study, the target scope of special education domain is three types of specific learning disabilities; dyslexia, dyscalculia and dysgraphia. In specific learning disabilities field, children that may have potential for specific learning disabilities should be identified in early stage in order to assist their social and academic's development. The identification process is very complicated and currently it is manually done by experts. Children suspected with specific learning disabilities have to undergo a thorough checking before specifically categorizing them into dyslexia, dysgraphia or dyscalculia. Therefore, the purposes of developing special education ontology model are to support decisions in early identification and intervention's recommendation. There are several required lists to be used as the references for identification purposes. The lists are characteristics of dyslexia, dysgraphia and dyscalculia, and several related intervention and rehabilitation activities for each type. The output of this process is a set of natural language questions, called as competency question. Competency questions were used to represent the ontology requirements. An excerpt of competency questions for developing the ontology model is listed in Table 1.

Table 1. An excerpt of competency questions

\begin{tabular}{cl}
\hline No. & Question \\
\hline 1. & What are the characteristics of student that have a learning problem? \\
2. & Is a student that have learning problem got the same characteristics with student that have specific learning disability? \\
3. & What are the characteristics of student that have specific learning disability? \\
4. & Are there categories in specific learning disability? \\
5. What are the characteristics of student that has dyslexia? \\
6. What are the characteristics of student that has dysgraphia? \\
7. What are the characteristics of student that has dyscalculia? \\
8. If the children have only one characteristic of dyslexia, is the student might have dyslexia? \\
9. If the children have only one characteristic of dysgraphia, is the student might have dysgraphia? \\
10. If the children have only one characteristic of dyscalculia, is the student might have dyscalculia?
\end{tabular}

\subsection{Build glossary of terms}

Glossary of terms is the documentation of all the relevant concepts and relations between concepts of special education domain, their descriptions, synonyms and acronyms. All these terms were identified from the obtained answers of competency questions. The special education ontology comprised of five basic concepts characteristics, activity, lexiaCharac, graphiaCharac and calculiaCharac. These concepts are inherited from top-level concept of Thing. Concept of characteristics is about the characteristic of special education in term of learning problem. The characteristics is a feature belonging typically to a person that might have a learning problem that serving to identify them. Based on the feature that have, they can be identified. Meanwhile concept of activity is about an activity that specific learning disability children can commit. It is a specific activity that help those children to learning and practice to make a good life in the future. Each type of activity is linked to each type of problem in learning. Concept of lexiaCharac is about characteristic of dyslexia that mainly have a problem in reading. Whilst, the concept graphiaCharac is about characteristic of dyslexia that mainly have a problem in writing. Concept of calculiaCharac is about characteristic of dyslexia that mainly have a problem in mathematics.

\subsection{Build concept taxonomies}

There are three types of conceptual taxonomies that have been defined in the ontology model; subclass-of, exhaustive-decomposition and partition. Figure 2 shows the subclass-of relations between concepts. Subclass refers to the concept which is derived from another concept. For example, the concept of characteristic is a subclass of Thing, meanwhile the concept of learningProblem is derived from the concept of characteristic.

The concepts of lexiaCharac, graphiaCharac, and calculiaCharac make up an exhaustive decomposition of the concept characteristic as depicted in Figure 3. The concept of characteristic can be decomposed exhaustively into the concepts of lexiaCharac, graphiaCharac, and calculiaCharac because there are no instances of the concept of characteristic that are not instances of one of these three concepts. Also, the instance in the concept of lexiaCharac can be in the concept of graphiaCharac or calculiaCharac. 
Figure 4 shows that the concept of activity and characteristic make up the partition in relation to the concept of owl: Thing because the concept of activity and characteristic are a set of mutually disjointed classes and do not share common instances. Every instance of the concept of characteristic is an instance of exactly one of the subclasses in the partition.

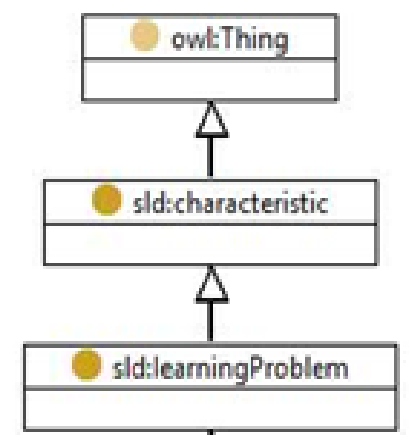

Figure 2. Subclass-of relation

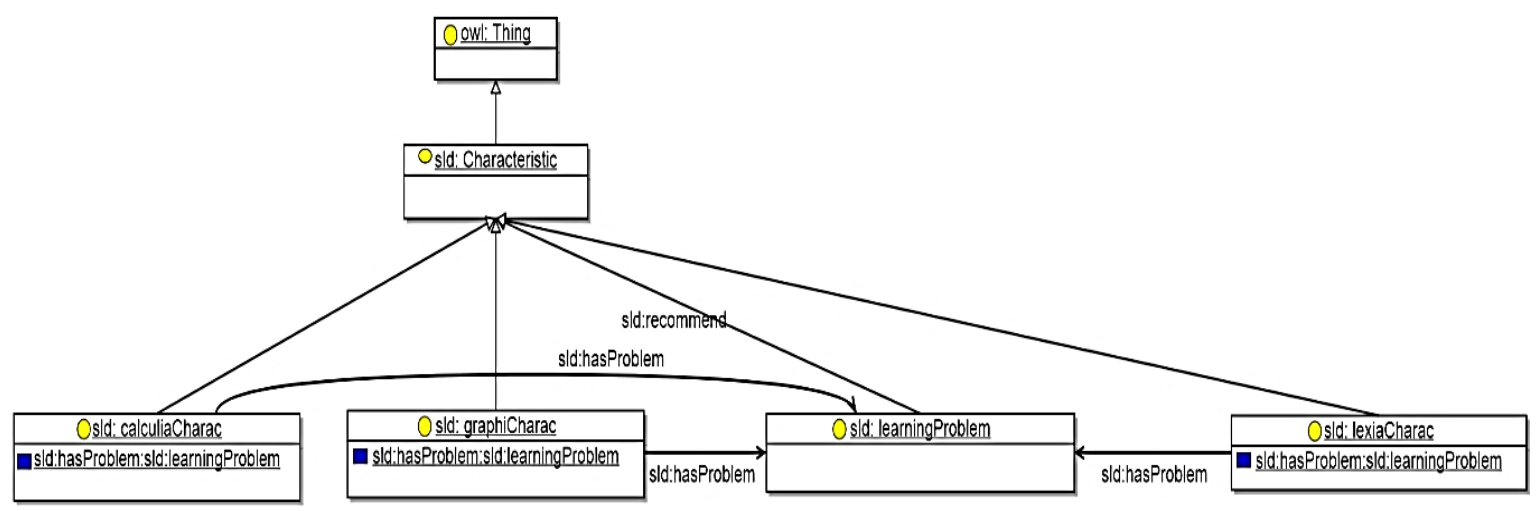

Figure 3. Exhaustive-decompositon relation

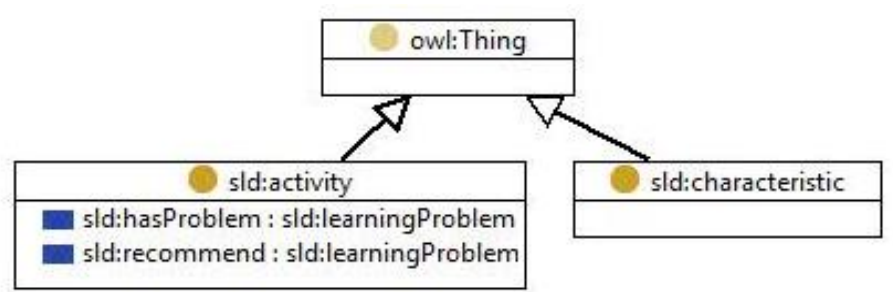

Figure 4. Partition relation

\subsection{Build ad hoc relation diagram}

Once the conceptual taxonomy has been constructed, ad hoc relationships between concepts are built. Figure 5 depicts a fragment of the ad hoc binary relation diagram of the ontology model, with the relation of hasProblem between the concepts of graphiaCharac and learningProblem. The domain and ranges of each argument of each relation delimit exactly and precisely the classes that are appropriate for the relation.

\begin{tabular}{|c|c|c|}
\hline Osld: graphiCharac & & Os sld: learningProblem \\
\hline 口 sld:hasProblem:sidilearningProblem & sld:hasProblem & \\
\hline
\end{tabular}

Figure 5. An excerpt of the diagram of ad hoc binary relation of ontology model 


\subsection{Build concept dictionary}

A concept dictionary includes all the domain concepts and their relations. Table 2 shows an excerpt of concept dictionary of ontology model.

Table 2. An excerpt of the concept dictionary of the ontology model

\begin{tabular}{cc}
\hline Concept name & Relations \\
\hline activity & recommend \\
characteristics & - \\
calculiaCharac & hasProblem \\
lexiaCharac & hasProblem \\
graphiaCharac & hasProblem \\
learningProblem & recommend \\
calculation & hasProblem \\
comprehension & - \\
\hline
\end{tabular}

\subsection{Describe ad hoc binary relation}

The relation of hasProblem means that the children who are having calculiaCharac (dyscalculia characteristic), graphiaCharac (dysgraphia characteristic) and lexiaCharac (dyslexia characteristic), are considered to have a problem in learning, that is in the concept of learningProblem. It can be a reverse relation, when the children have a problem in learning (learningProblem), it is a problem of calculiaCharac (dyscalculia characteristic) or graphiaCharac (dysgraphia characteristic) or lexiaCharac (dyslexia characteristic). The descriptions of ad hoc binary relations defined in the ontology is shown in Table 3.

Table 3. The ad hoc binary relation table of ontology

\begin{tabular}{ccccc}
\hline Relation name & Source concept & Source card (Max) & Target concept & Inverse relation \\
\hline sld:hasProblem & $\begin{array}{l}\text {-calculiaCharac } \\
\text {-graphiaCharac } \\
\text {-lexiaCharac }\end{array}$ & 1 & learningProblem & is a problem of \\
\hline
\end{tabular}

\subsection{Describe formal axioms}

Class axioms are identified and an example of the expression are shown as in Figure 6 for describing any children with dyscalculia usually have problems related to mathematics such as recognition of numbers, calculations and measurements.

\begin{tabular}{|c|c|}
\hline Axiom name & Dyscalculia characteristic \\
\hline Description & Every characteristics that is dyscalculia characteristics that has problem in learning \\
\hline Expression & $\begin{array}{l}\text { If } \\
\text { there is some problem with calculation } \\
\text { Then } \\
\text { it is a dyscalculia characteristics } \\
\text { End if } \\
\text { If } \\
\text { there is some problem with calendar } \\
\text { Then } \\
\text { it is a dyscalculia characteristics } \\
\text { End if } \\
\text {... }\end{array}$ \\
\hline Concept & $\begin{array}{l}\text { characteristic, calculiaCharac, calculation, calendar, comprehension, counting, measures, } \\
\text { memory numbers, numbers, spatialTemporal and word }\end{array}$ \\
\hline $\begin{array}{l}\text { Ad hoc binary } \\
\text { relation }\end{array}$ & hasProblem \\
\hline
\end{tabular}

Figure 6. An excerpt of formal axioms expression for class dyscalculia characteristics

\subsection{Describe rules}

A set of rules are identified to be inferred. Here, rules are describes to include the informations such as rule name, it's description in natural language form, the expression of the rule, concepts that involve, variables and relation. Figure 7 shows an example of a rule constructed in the ontology model and Figure 8 demontrates the result inferred. 


\begin{tabular}{|c|l|}
\hline Rule name & Screening rule \\
\hline Description & Every characteristics that is dyscalculia characteristics that has problem in learning \\
\hline Expression & If comprehension(?a) and counting(?b) and [hasProblem](?a)(?b) then [calculiaCharac] \\
\hline Concept & Comprehension, Counting, calculiaCharac \\
\hline Ad hoc binary relation & hasProblem \\
\hline Variables & $? \mathrm{a}$ \\
& $? \mathrm{~b}$ \\
\hline
\end{tabular}

Figure 7. The rule for identification of potential children with specific learning disabilities

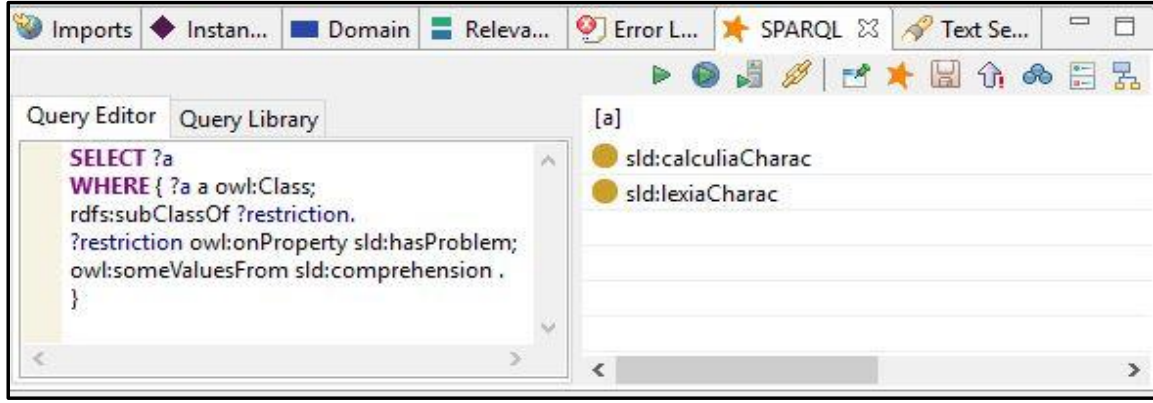

Figure 8. Type of specific learning disabilities identified based on rule inferred

\section{CONCLUSION}

This research focuses on the development of a new ontology model in special education domain. This model is used to capture the semantics of knowledge for the purposes of providing early identification of children with specific learning disabilities. The proposed ontology model specifically designed for the identification of three types of learning disabilities: dyslexia (reading difficulties), dyscalculia (mathematical related problems) and dysgraphia (writing problems). The types of disabilities can be determined based on their characteristics and appearances, which were included in the database of the ontology model. The ontology model proposed here employed a rule-based approach which allows the inference of new knowledge based on the patterns of existing knowledge.

The first major contribution of this study in terms of its knowledge engineering is the development of a new ontology that models the semantics of knowledge in specific learning disabilities, which serve as a source of knowledge for supporting various decision-making activities in special education environment. The main benefit of this ontology model lies in it to support decisions-makings in early screening of children with learning problem and recommendation of educational intervention activities based on the identified problem. Ontology is built on OWL semantic language, and not only benefits experts from different domain, but also general users in having common understanding and mutual knowledge sharing. Any experts can reuse and expand this domain ontology for other categories of special education for achieving similar task. In addition, ontology developer can use this domain ontology to build similar domain ontology or application ontology in the area of special education.

\section{ACKNOWLEDGEMENTS}

This research is sponsored by the Ministry of Higher Education, under the Fundamental Research Grants Scheme vote 59395.

\section{REFERENCES}

[1] Y. Lv, et al., "Multi-Level Ontology Integration Model for Business Collaboration," Int. J. Adv. Manuf. Technol., vol. 84, pp. 445-451, 2016.

[2] Y. Kassahun, et al., "Automatic Classification of Epilepsy Types using Ontology-Based and Genetics-Based Machine Learning," Artif. Intell. Med., vol. 61, pp. 79-88, 2014.

[3] A. Frid, et al., "Computational Diagnosis of Parkinson's Disease Directly from Natural Speech Using Machine Learning Techniques,” 2014 IEEE Int. Conf. Softw. Sci. Technol. Eng., vol. 1, pp. 50-53, 2014.

[4] J. M. Alja 'am, et al., "An Assistive Computerized System for Children with Intellectual and Learning Disabilities," LNCS, vol. 5597, pp. 9-16, 2009. 
[5] J. M. Aljaam, et al., "An Ontology-Based System to Dynamically Extract Multimedia Elements for Children's Tutorials, ” 2012 2nd International Conference on Advances in Computational Tools for Engineering Applications (ACTEA), pp. 29-32, 2012.

[6] J. Schoenfisch, et al., "Root Cause Analysis in IT Infrastructures using Ontologies and Abduction in Markov Logic Networks," Inf. Syst., vol. 74, pp. 103-116, 2016.

[7] C. Guangzuo, et al., "OntoEdu: A Case Study of Ontology-Based Education Grid System for E-Learning," Global Chinese Conference on Computers in Education 2004, pp. 6-10, 2004.

[8] A. Lalingkar and C. R. S. Ramani, "Ontology-based Smart Learning Environment for Teaching Word Problems in Mathematics," Lecture Notes in Educational Technology, pp. 251-258, 2015.

[9] H. S. Chung and J. M. Kim, "Ontology Design for Creating Adaptive Learning Path in e-Learning Environment," Proceedings of the International Multi Conference of Engineers and Computer Scientists (IMECS 2012), vol. 1, pp. 585-588, 2012.

[10] H. Kook, et al., "Multi-Stimuli Multi-Channel Data and Decision Fusion Strategies for Dyslexia Prediction using Neonatal ERPs," Pattern Recognit., vol. 38, pp. 2174-2184, 2005.

[11] A. Kalou, et al., "An Ontology Model for Building, Classifying and Using Learning Outcomes," 2012 IEEE 12th Int. Conf. Adv. Learn. Technol., pp. 61-65, 2012.

[12] V. Luna, et al., "An Ontology-Based Approach for Representing the Interaction Process between User Profile and Its Context for Collaborative Learning Environments," Comput. Human Behav., vol. 51, pp. 1387-1394, 2015.

[13] M. Peleg, et al., "Onto-clust-A methodology for Combining Clustering Analysis and Ontological Methods for Identifying Groups of Comorbidities for Developmental Disorders," J. Biomed. Inform., vol. 42, pp. 165-175, 2009.

[14] S. Cramerotti and D. Ianes, "An Ontology-based System for Building Individualized Education Plans for Students with Special Educational Needs,” Procedia -Social Behav. Sci., vol. 217, pp. 192-200, 2016.

[15] J. T. Nganji and M. Brayshaw, "Designing and Reflecting on Disability-Aware E-learning Systems: The Case of ONTODAPS, ” 2014 IEEE 14th Int. Conf. Adv. Learn. Technol., pp. 571-575, 2014.

[16] J. T. Nganji and S. H. Nggada, "Disability-Aware Software Engineering for Improved System Accessibility and Usability," Int. J. Softw. Eng. its Appl., vol. 5, pp. 47-62, 2011.

[17] K. Venkatesan, et al., "Hybrid ontology based e-Learning expert system for children with Autism," 2013 International Conference of Information and Communication Technology, ICoICT 2013, pp. 93-98, 2013.

[18] C. Cortiella and S. H. Horowitz, "The state of learning disabilities: Facts, trends and emerging Issues," Natl. Cent. Learn. Disabil., vol. 3, pp. 1-52, 2014.

[19] E. G. Willcutt, et al., "Colorado Learning Difficulties Questionnaire:Validation of a parent-report screening measure," Psychol. Assess., vol. 23, pp. 778-791, 2011.

[20] H. M. Ekhsan, et al., "The Implementation of Interactive Multimedia in Early Screening of Dyslexia,” International Conference on Innovation, Management and Technology Research, pp. 566-569, 2012.

[21] K. N. Elsayed, "Diagnosing Learning Disabilities in a Special Education by an Intelligent Agent Based System," 4th Comput. Sci. Electron. Eng. Conf., pp. 7-12, 2012.

\section{BIOGRAPHIES OF AUTHORS}

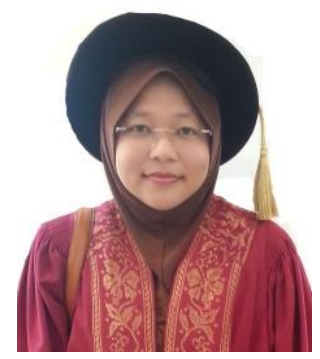

Rosmayati Mohemad is currently a senior lecturer at Faculty of Ocean Engineering Technology and Informatics, Universiti Malaysia Terengganu (UMT). She received her PhD degree in Computer Science from National University of Malaysia in 2013. Her research interests are in knowledge engineering and decision support system and currently focusing on modelling ontology for supporting decision-making process in the domains of special education and forensics science. She is an editor of books, conference proceedings and also reviewer of international journals and conference papers. She is a profesional technologist of Malaysian Board of Technologies and also a member of IEEE society.

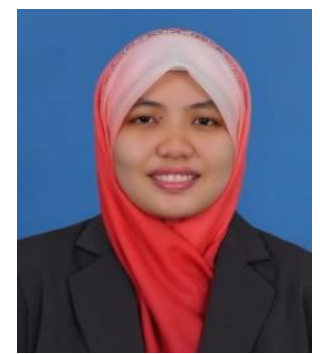

Nur Fadila Akma Mamat is currently a postgraduate student at Faculty of Ocean Engineering Technology and Informatics, Universiti Malaysia Terengganu (UMT). Her recent study focuses on modeling ontology model in special education domain. She has published several international journals and presented papers at various internal and local conferences. 

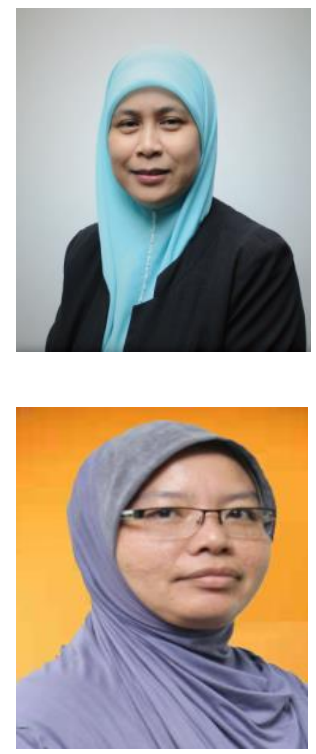

Noor Maizura Mohamad Noor is currently a professor at Faculty of Ocean Engineering Technology and Informatics, Universiti Malaysia Terengganu (UMT). Her recent research work focuses on improving organizational decision-making practices through the use of technologies. This includes research interests in the design, development and evaluation of decisionsupport systems for analyzing and improving decision processes. Her research interests also focus on the areas of computer science, intelligent decision support system, clinical decision support system, and information system as well as action research in education. She has presented and published over two hundred of research papers on the decision support system at various international and local refereed journals, conferences, seminars and symposiums.

Arifah Che Alhadi is currently a senior lecturer at Faculty of Ocean Engineering Technology and Informatics, Universiti Malaysia Terengganu (UMT). She received her Master degree in Information Technology from National University of Malaysia in 2005. Her recent research work focuses on Information Retrieval especially in the short text analysis and retrieval. Besides that, she also involved in the research on ontology development and sentiment analysis. She is actively conducting her research in her field of interests through the supervision of undergraduate students. She is also actively involved as paper reviewers for national and international journals, conferences, seminars and symposiums in her field. 